\title{
OPTIMIZATION OF AN OPERATING POINT IN HIGH INTENSITY SYNCHROTRONS
}

\author{
S. Machida, KEK, Ibaraki-ken, Tsukuba-shi, 305-0801 Japan \\ shinji.machida@kek.jp
}

\begin{abstract}
In a synchrotron such as JHF $3 \mathrm{GeV}$ proton synchrotron (PS) and $50 \mathrm{GeV}$ PS, choice of an operating point in tune space depends mostly high intensity behavior of a proton beam, not by error fields of magnet manufacturing. Taking that into account, we have started a dry run of optimisation of an operating point, namely simulation of tune survey with space charge effects. The idea is to simulate the early stage of commissioning process so that obviously fatal area in tune space is excluded from the beginning in the real commissioning and unnecessary radio-activation due to beam loss is minimized.
\end{abstract}

\section{INTRODUCTION}

In a next generation proton accelerator such as JHF synchrotron and SNS storage ring, one or two order more number of particles is expected to realize beam power of $1 \mathrm{MW}$ and higher. Although careful choice of lattice and beam parameters is made as much as possible during the design stage, eventually a lot of machine tuning and beam study using the real machine are needed to reach the design goal. From the previous experiences of existing synchrotrons, we have ideas how we tune a machine and what kinds of beam study are necessary to improve beam quality.

Having said that, it should be, however, noted that all the procedures based on the previous experiences may not be performed because of the limitation of radio-activation. For example, with full intensity operation, machine parameters cannot be chosen so that more than $10 \%$ of the particles are lost, simply because such as beam loss is prohibited. In that respect, a dry run of commissioning and machine improvement becomes more serious compared with the existing facilities.

Needless to say, the computation power is still a big issue to perform a dry run. Although the CPU power becomes incredibly faster than the one, say 10 years ago, the reproduction of a real machine in a computer is not possible. Furthermore, the most crucial aspect of the beam behaviour is determined by multi-particles, which interact continuously. We need to focus on some phase of machine operation, such as a injection energy region. We will discuss the optimisation of operating point as an example.

\section{MODEL}

As a simulator, the code Simpsons [1] is employed. In order to speed up the tracking, 2D version of the code is used, which models the peak line density of the beam at injection energy without acceleration process. We observed the rms emittance at 1,000 turns after injection. It takes around 2.5 hour CPU time for each run with an Alpha workstation. Simulation of a multi-turn injection is omitted, which is discussed in another paper using 3D version of the code [2].

As a test lattice, JHF $3 \mathrm{GeV}$ PS is used. No magnet errors are included. The beam intensity and initial emittance assumed in the simulation are similar to that of the $3 \mathrm{GeV}$ PS, namely $30 \mathrm{~A}$ at peak and $36.5 \pi \mathrm{mm}-\mathrm{mrad}$ for both transverse planes, respectively. The emittance value quoted above is normalized rms one. The charge distribution is waterbag. Momentum spread of $0.2 \%$ (rms) is included with parabolic distribution. With those beam parameters, the incoherent direct tune shift is -0.32 .

The $3 \mathrm{GeV}$ PS lattice has three-fold symmetry so that the structure resonance lines up to $4^{\text {th }}$ order is depicted in Fig. 1. We have explored on the tune space horizontally and vertically. The test bare tunes are indicated as dots in the same figure. The nominal bare tune is $(7.35,5.80)$.

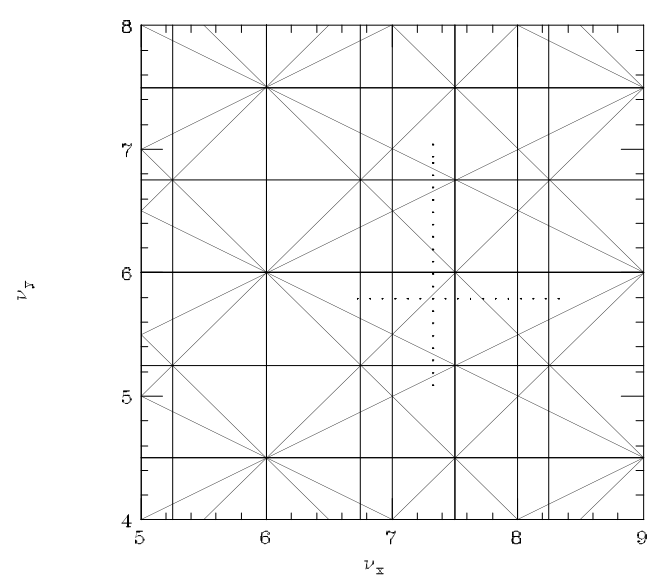

Figure 1: Tune diagram of $3 \mathrm{GeV}$ PS. The dots shows the bare tune we have explored. The nominal tune is $(7.35,5.80)$

\section{RESULTS}

\subsection{Horizontal Tune Survey}

Figure 2 shows the rms emittance as a function of horizontal bare tune. The vertical bare tune is fixed at $v y=5.8$. Taking the tune shift into account, some peaks indicating emittance growth can be attributed to structure resonances. A peak at $v x=6.95$ is due to $4 v x=27$, one at $v x=7.75$ is due to $2 v x=15$ or $4 v x=30$, and one at $v x=8.15$ 
is due to $2 v x+2 v y=27$. A small peak at $v x=7.25$ may be due to $2 v x-2 v y=3$.

- horizontal

- vertical

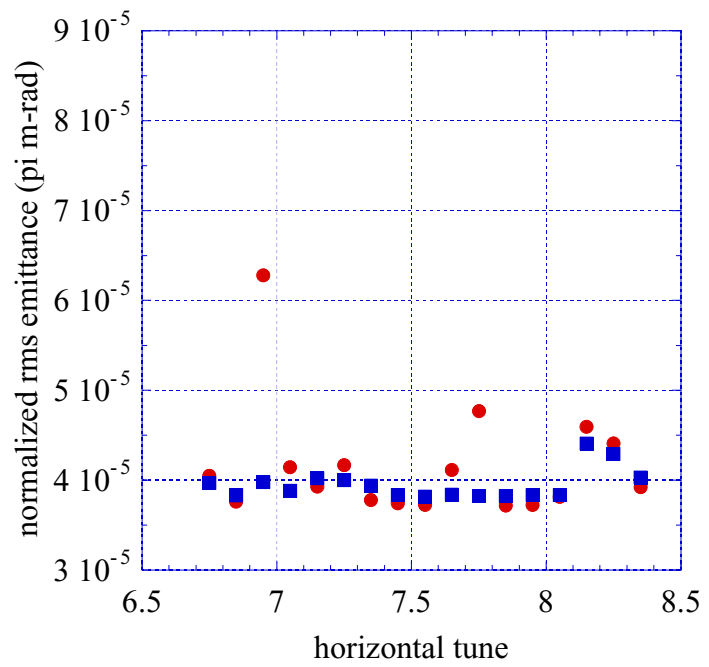

Figure 2: RMS emittance at 1,000 turns after injection when bare tune is surveyed in horizontal direction.

In addition to the rms emittance, we estimated beam loss assuming there is an aperture limit at radius of $0.150 \mathrm{~m}$ all around the ring. In fact, that aperture limit is rather large compared with the real machine so that the beam loss estimate here is more optimistic. Figure 3 shows that there is slight beam loss associated with the emittance growth at $v \mathrm{x}=7.75$.

- survival

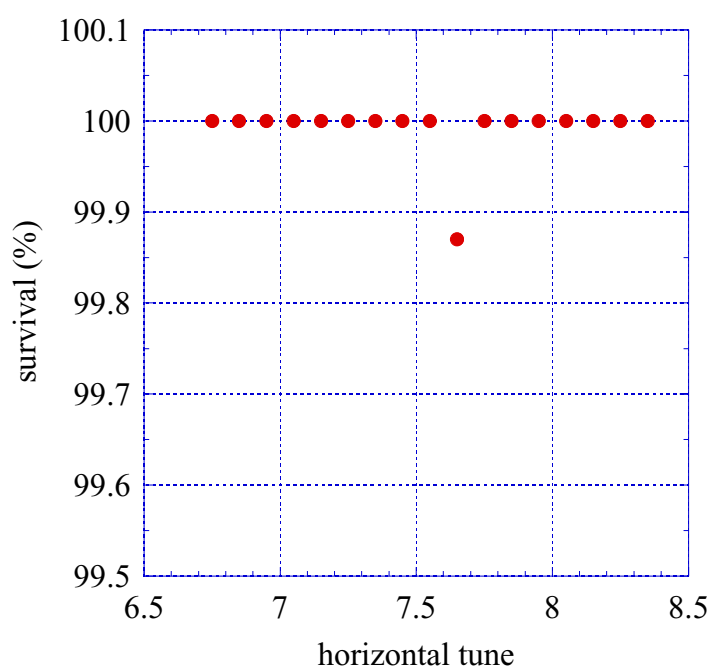

Figure 3: Beam survival at 1,000 turns after injection when bare tune is surveyed in horizontal direction.

\subsection{Vertical Tune Survey}

Similarly, tune survey in vertical direction was carried out and the results are shown in Fig. 4. Some peaks are identified as follows. A peak at $v y=6.20$ is due to $4 v y=24$, one at $v y=6.95$ is due to $4 v y=27$, one at $v y=6.60$ is due to $2 v x+2 v y=27$, and one at $v y=5.50$ is due to $4 v y=21$. A small peak at $v y=5.70$ may be due to $2 v x-2 v y=3$.

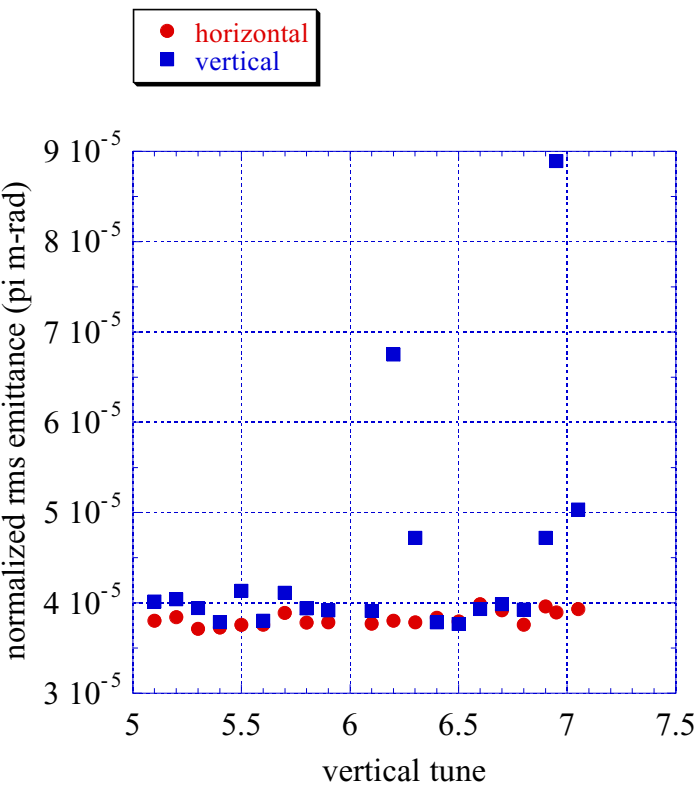

Figure 4: RMS emittance at 1,000 turns after injection when bare tune is surveyed in vertical direction.

We also estimated beam loss. Figure 5 shows that there is slight beam loss associated with the emittance growth at $v y=6.20$ and $v y=6.95$.

- survival

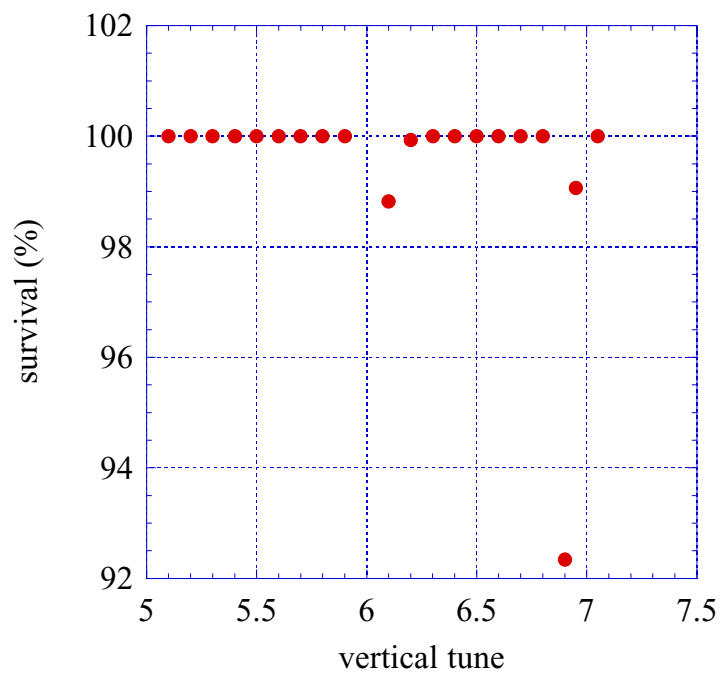

Figure 5: Beam survival at 1,000 turns after injection when bare tune is surveyed in vertical direction. 


\section{DISCUSSIONS}

From this simulation, we concluded that the nominal bare tune at $(7.35,5.80)$ should be a good starting point. We probably examine bare tune just below $v \mathrm{x}=8$ in horizontal direction or just above $v \mathrm{y}=6.5$ in vertical as an alternative. Other area should be excluded for survey in the real machine.

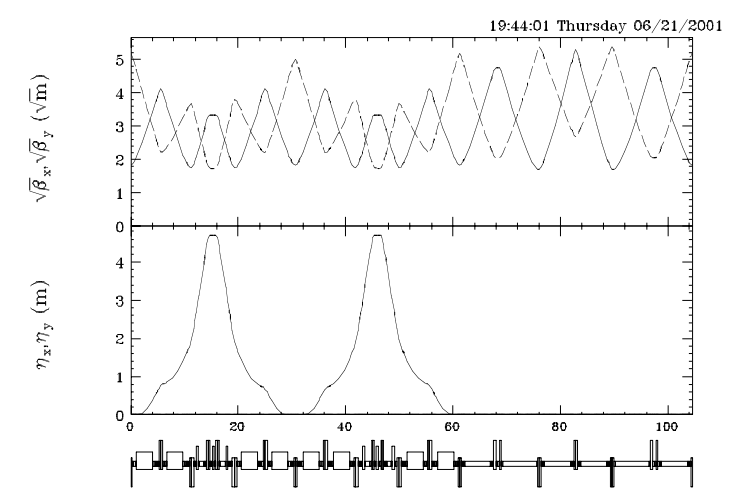

Figure 6: Lattice function of $3 \mathrm{GeV}$ PS. One superperiod is shown. Solid line is horizontal beta function and dashed line is vertical.
Apart from the primary purpose of finding the optimum operating point, one thing we noticed from this simulation is the strong harmonic component of 27. In both horizontal and vertical directions, large emittance growth is observed at $v x, y=6.95$. As shown in Fig. 6, 3GeV PS lattice has primarily $27^{\text {th }}$ beta modulation. The harmonic of 27, in fact, corresponds to 90 degree phase advance as an average although it is not the case locally. Whether this explains the large emittance growth needs further study.

\section{REFERENCES}

[1] S. Machida, et. al., "Simulation of Space Charge Effects in a Synchrotron", AIP Conf. Proc. 448, p.73.

[2] I. Sakai, et. al., "Intensity dependent emittanceexchange in a high intensity proton synchrotron", in this proceedings. 